\title{
Docosahexaenoic acid is selectively enriched in plasma phospholipids during pregnancy in Trinidadian women - Results of a pilot study
}

\author{
Graham C. BURDGE ${ }^{a *}$, Rachel C. SHERMAN ${ }^{\mathrm{a}}$, Zulaika ALI ${ }^{\mathrm{b}}$, \\ Stephen A. WOOTTON ${ }^{\mathrm{a}}$, Alan A. JACKSON ${ }^{\mathrm{a}}$ \\ ${ }^{a}$ Institute of Human Nutrition, Biomedical Sciences Building, University of Southampton, \\ Bassett Crescent East, Southampton, SO16 7PX, UK \\ $\mathrm{b}$ The University of the West Indies, St. Augustine, Trinidad and Tobago
}

(Received 9 May 2005; accepted 31 August 2005)

\begin{abstract}
The fetal demand for docosahexaenoic acid (DHA) has to be satisfied by the mother. We determined the fatty acids in maternal plasma non-esterified fatty acid (NEFA), triacylglycerol (TAG) and phosphatidylcholine (PC), in a cross-sectional study of non-pregnant $(n=10)$, pregnant $(n=19)$, and postpartum $(n=9)$ women. There were lipid class-dependent differences in plasma polyunsaturated fatty acid (PUFA) concentrations between groups. During pregnancy, DHA was most highly enriched in PC, about $230 \%$, with more modest enrichment for linoleic acid (LA) and arachidonic acid (AA), and no enrichment of alpha-linolenic acid ( $\alpha$-LNA). There was relative enrichment of LA, AA and $\alpha$-LNA in TAG, but not of DHA. There was no specific enrichment of any PUFA in the NEFA pool. These data accord with the suggestion that the enrichment of $\alpha$-LNA in TAG and of DHA in phospholipids reflects hepatic regulation of n-3 PUFA metabolism which potentially enhances the delivery of DHA to the placenta.
\end{abstract}

pregnancy / human / plasma / polyunsaturated fatty acid

\section{INTRODUCTION}

The fetus has a high demand for the n-3 polyunsaturated fatty acid (PUFA) docosahexaenoic acid (22:6n-3, DHA), which accumulates in the phospholipids of neural membranes of the developing central nervous system. Insufficient accumulation of DHA is associated with impaired cognitive and visual function $[1,2]$. The fetus is dependent upon a maternal supply of preformed DHA since it has a limited capacity for synthesising DHA from its precursor, $\alpha$ linolenic acid ( $\alpha$-LNA) [3].
It has been reported that in human pregnancy, the concentration of DHA in plasma phospholipids increases, specifically $s n-1$ palmitoyl, sn-2 docosahexaenoyl phosphatidylcholine (PC) [4-7], although not all studies have found this [8]. It has been assumed that this increase is important for the delivery of DHA to the fetus [4-7]. We wished to know whether other plasma lipid pools were similarly enriched in DHA: nonesterified fatty acid (NEFA) derived from adipose tissue, and triacylglycerol (TAG) which derive from liver metabolism. We report the results of a pilot cross-sectional

\footnotetext{
* Corresponding author: g.c.burdge@ soton.ac.uk
} 
study of the PUFA composition of individual plasma lipid pools in mid and late pregnancy, and within 24 hours of birth.

\section{MATERIALS AND METHODS}

The study was conducted at the Mount Hope Maternity Hospital, Trinidad. Ethical approval was granted and informed consent was obtained. Subject details are summarised in Table I. Fasting blood samples were collected from non-pregnant staff, women attending an antenatal clinic with uncomplicated pregnancies in mid (20-24 weeks) and late (30-34 weeks) gestation, and women on the morning after delivery. Plasma was isolated by centrifugation and stored at $-20{ }^{\circ} \mathrm{C}$. Non-pregnant women had no documented pregnancy and all pregnant women were primigravid.

Total lipids were extracted from plasma $(1.0 \mathrm{~mL})$ with chloroform/ methanol 2:1 (v/v) [9]. Plasma PC, TAG and NEFA fractions were isolated by solid phase extraction, converted to fatty acid methyl esters and analysed by gas chromatography [10].

Data are presented as $\mu \mathrm{mol} \cdot \mathrm{L}^{-1}$ plasma, mean \pm standard deviation. Statistical comparisons between groups of women were done by one-way ANOVA with the Bonferroni post hoc test.

\section{RESULTS}

The most marked differences were for plasma PC. DHA was significantly greater in the PC of pregnant and postpartum women than for non-pregnant women (about 230\%) (Tab. I). Linoleic acid (LA) in PC was significantly greater in mid and late pregnancy than in the non-pregnant $(170 \%$ and $150 \%$, respectively) or in the postpartum group $(130 \%$ and $110 \%$, respectively). Arachidonic acid (AA) in PC was consistently greater in pregnant women and the postpartum group (about $160 \%$ ) than in non-pregnant women.
For the NEFA fraction, there were no significant differences in the concentrations of the n-6 PUFA, LA, or the n-3 PUFA, alpha-linolenic acid ( $\alpha$-LNA) and DHA amongst groups. Although the n- 6 PUFA AA was measured in plasma NEFA in non-pregnant women, it was undetectable in plasma NEFA of the pregnant and postpartum groups.

For plasma TAG, in pregnancy there were higher concentrations of LA (about $150 \%)$, AA (about 180\%) and $\alpha$-LNA (190\%) compared with non-pregnant or postpartum women. There were no significant differences between groups in DHA concentration in TAG.

The total lipid concentrations in plasma from these women have been reported previously. The differences were modest with TAG concentrations being about $60 \%$ higher during pregnancy and no demonstrable difference in NEFA or PC [11].

\section{DISCUSSION}

We found that during pregnancy the pattern of fatty acids varies widely among different lipid pools in plasma. For n-3 PUFA, the most marked change was relative enrichment of DHA, but not $\alpha$-LNA, in PC. This compares with relative enrichment of $\alpha$-LNA, but not DHA, in TAG. For n-6 PUFA the changes were less marked, with relative increases in LA and AA in both TAG and PC. There were no demonstrable differences for any of these fatty acids in plasma NEFA. Together these data represent a significant extension in the understanding of the regulation of fatty acid metabolism during pregnancy beyond previous studies which have focussed on single lipid classes or total plasma lipids [4-8].

Fatty acids in maternal circulation are potentially available for uptake by the placenta to meet the needs of the fetus. The pattern of enrichment of individual fatty acids within different plasma lipid pools is likely to reflect the intracellular compartment 


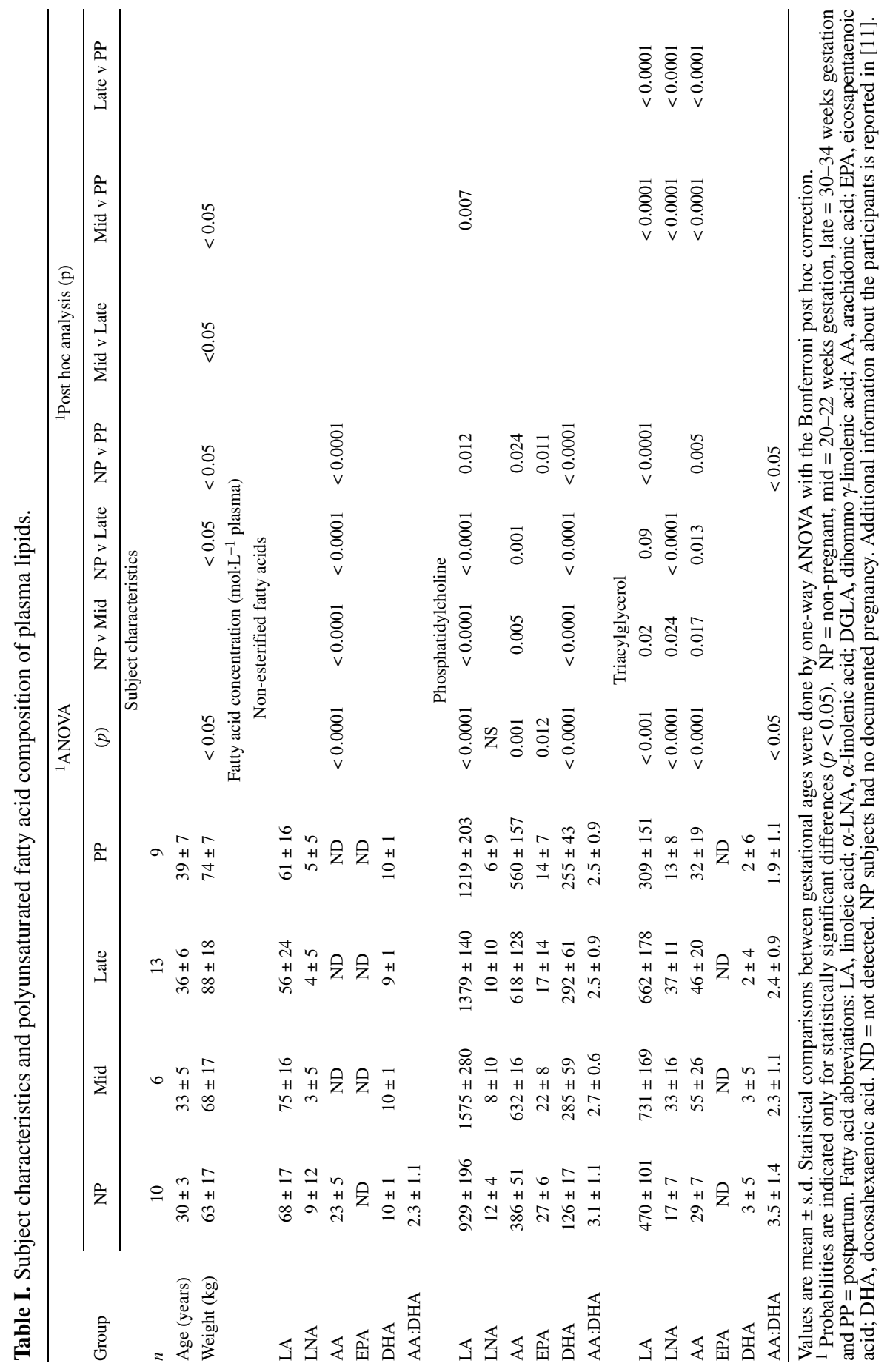


from which they are derived and may enable differential delivery to the fetus. Fasting plasma NEFA reflect adipose tissue, while the TAG and PC fractions, primarily associated with VLDL, reflect intra-hepatic lipid biosynthesis and secretion. Plasma NEFA are precursors for synthesis of VLDL by the liver in pregnancy [12]. The fatty acid composition of plasma NEFA was not different during pregnancy and provided no indication of selective mobilisation of individual fatty acids from the hydrolysis of TAG in adipose tissue, although this mobilisation may be important for the bulk supply of fatty acids to the liver [12]. By contrast, there were important differences in the fatty acid profiles of plasma TAG and PC, suggesting selective utilisation of individual fatty acids in their formation. These data indicate that although dietary intake may be an important determinant of the amount of individual fatty acids in plasma, the plasma pool in which they are incorporated and the changes in concentration during gestation are determined by the specificity of maternal hepatic lipid metabolism. This may have important implications for the bioavailability of individual fatty acid species.

The mechanisms which underlie these selective changes and their regulation during pregnancy are not clear. However, evidence for selective adaptations as an integral response to pregnancy suggests a mechanism through which individual PUFA might be supplied preferentially to the fetus. Fatty acids of the n- 6 series were enriched in plasma TAG and PC during pregnancy, but for the n-3 series, enrichment of $\alpha$-LNA was only found in TAG, and enrichment of DHA only found in PC. Previous evidence of specific enrichment of plasma $s n-1$ palmitoyl, $s n-2$ docosahexaenoyl PC in pregnancy [4], suggests that $s n-1$ palmitoyl PC species are preferentially hydrolysed by placental lipoprotein lipase [13]. Therefore specific enrichment of DHA in PC is likely to increase the bioavailability of this fatty acid to the placenta and fetus [14].
Despite the limitations of the cross sectional design, the results of this pilot study indicate the need to determine how maternal hepatic metabolism of individual fatty acids is regulated during pregnancy and how this relates to fetal development. It may be that the enrichment of $\alpha$-LNA in plasma TAG marks an intra-hepatic pool which is the precursor for DHA formation in PC.

\section{ACKNOWLEDGEMENTS}

We wish to thank Mr P Wright for assisting the analysis of the samples and Dr K Lutchman Singh for help with the recruitment of subjects and with the collection of samples.

\section{REFERENCES}

[1] Innis SM. Perinatal biochemistry and physiology of long-chain polyunsaturated fatty acids. J Pediatr 2003, 143 (Suppl): S1-S8

[2] Larque E, Demmelmair H, Koletzko B. Perinatal supply and metabolism of long-chain polyunsaturated fatty acids: importance for the early development of the nervous system. Ann NY Acad Sci 2002, 967: 299-310.

[3] Salem N, Wegher B, Mena P, Uauy R. Arachidonic and docosahexaenoic acids are biosynthesized from their 18-carbon precursors in human infants. Proc Natl Acad Sci USA 1996, 93: 49-54.

[4] Postle AD, Al MD, Burdge GC, Hornstra G. The composition of individual molecular species of plasma phosphatidylcholine in human pregnancy. Early Hum Dev 1995, 43: 47-58.

[5] Otto SJ, Houwelingen AC, Antal M, Manninen A, Godfrey K, Lopez-Jaramillo P, Hornstra G. Maternal and neonatal essential fatty acid status in phospholipids: an international comparative study. Eur J Clin Nutr 1997, 51: 232-242.

[6] Otto SJ, van Houwelingen AC, Badart-Smook A, Hornstra G. Changes in the maternal essential fatty acid profile during early pregnancy and the relation of the profile to diet. Am J Clin Nutr 2001, 73: 302-307.

[7] Al MD, van Houwelingen AC, Kester AD, Hasaart TH, de Jong AE, Hornstra G. Maternal essential fatty acid patterns during normal pregnancy and their relationship to the neonatal 
essential fatty acid status. Br J Nutr 1995, 74: 55-68.

[8] Matorras R, Ruiz JI, Perteagudo L, Barbazan MJ, Diaz A, Valladolid A, Sanjurjo P. Longitudinal study of fatty acids in plasma and erythrocyte phospholipids during pregnancy. J Perinat Med 2001, 29: 293-297.

[9] Folch JL, Lees M, Sloane-Stanley GH. A simple method for the isolation and purification of total lipids from animal tissues. J Biol Chem 1957, 226: 497-509.

[10] Burdge GC, Wright P, Jones AE, Wootton SA. A method for separation of phosphatidylcholine, triacylglycerol, non-esterified fatty acids and cholesterol esters from plasma by solid-phase extraction. Br J Nutr 2000, 84: 781-787

[11] Sherman RC, Burdge GC, Ali Z, Singh KL, Wootton SA, Jackson AA. Effect of preg- nancy on plasma lipid concentration in Trinidadian women. Result of a pilot study. West Indian Med J 2001, 50: 282-287.

[12] Herrera E. Lipid metabolism in pregnancy and its consequences in the fetus and newborn. Endocrine 2002, 19: 43-55.

[13] Samborski RW, Ridgway ND, Vance DE. Evidence that only newly made phosphatidylethanolamine is methylated to phosphatidylcholine and that phosphatidylethanolamine is not significantly deacylated-reacylated in rat hepatocytes. J Biol Chem 1990, 265: 1832218329.

[14] Ghebremeskel K, Crawford MA, Lowy C, Min Y, Thomas B, Golfetto I, Bitsanis D, Costeloe $\mathrm{K}$. Arachidonic and docosahexaenoic acids are strongly associated in maternal and neonatal blood. Eur J Clin Nutr 2000, 54: 50-56. 